\title{
Self-doped Mott insulator for parent compounds of nickelate superconductors
}

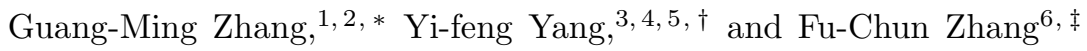 \\ ${ }^{1}$ State Key Laboratory of Low-Dimensional Quantum Physics and \\ Department of Physics, Tsinghua University, Beijing 100084, China \\ ${ }^{2}$ Frontier Science Center for Quantum Information, Beijing 100084, China \\ ${ }^{3}$ Beijing National Lab for Condensed Matter Physics and Institute of Physics, \\ Chinese Academy of Sciences, Beijing 100190, China \\ ${ }^{4}$ School of Physical Sciences, University of Chinese Academy of Sciences, Beijing 100190, China \\ ${ }^{5}$ Songshan Lake Materials Laboratory, Dongguan, Guangdong 523808, China \\ ${ }^{6}$ Kavli Institute for Theoretical Sciences and CAS Center for Topological Quantum Computation, \\ University of Chinese Academy of Sciences, Beijing 100190, China
}

(Dated: September 27, 2019)

\begin{abstract}
We propose the parent compound of the newly discovered superconducting nickelate $\mathrm{Nd}_{1-x} \mathrm{Sr}_{x} \mathrm{NiO}_{2}$ as a self-doped Mott insulator, in which the low-density Nd-5d conduction electrons couple to localized Ni-3 $d_{x^{2}-y^{2}}$ electrons to form Kondo spin singlets at low temperatures. This proposal is motivated with our analyses of the reported resistivity and Hall coefficient data in the normal state, showing logarithmic temperature dependence at low temperatures. In the strong Kondo coupling limit, we derive a generalized $t-J$ model with both Kondo singlets and nickel holons moving through the lattice of otherwise nickel spin- $1 / 2$ background. The antiferromagnetic longrange order is therefore suppressed as observed in experiments. With Sr-doping, the number of holons on the nickel sites increases, giving rise to the superconductivity and a strange metal phase analogous to those in superconducting copper oxides.
\end{abstract}

Introduction. - Recent discovery of superconductivity in $\mathrm{Nd}_{0.8} \mathrm{Sr}_{0.2} \mathrm{NiO}_{2}$ [1] has stimulated intensive interest in understanding its pairing mechanism, in particular, its similarity and difference compared to that in cuprate superconductors [2 7]. Despite tremendous efforts over past thirty years, high $T_{c}$ superconductivity (SC) remains one of the most challenging topics in condensed matter physics [8 11]. The parent compounds of copper oxides may be described as a Mott insulator with antiferromagnetic (AF) long-range order. Superconductivity arises when additional holes are introduced on the oxygen sites in the $\mathrm{CuO}_{2}$ planes upon chemical doping. These holes combine with the $3 d_{x^{2}-y^{2}}$ spins of $\mathrm{Cu}$-ions to form the Zhang-Rice singlets moving through the square lattice of $\mathrm{Cu}$-ions by the exchange with their neighboring $\mathrm{Cu}$-spins, which leads to an effective two-dimensional $t-J$ model to describe the low-energy physics of the cuprates [12]. The $\mathrm{AF}$ order is destroyed rapidly by small hole doping, while at optimal doping, the $d$-wave SC is established in bulk cuprates [13 15]. It has been a long-standing question if these "cuprate-Mott" conditions can be realized in other oxides. Extensive efforts have been made to investigate the nickel oxides both theoretically and experimentally [16 26].

Single crystal thin films of infinite-layer nickelates were lately synthesized using soft-chemistry topotactic reduction. Superconductivity was reported below $9 \sim 15 \mathrm{~K}$ in the hole-doped $\mathrm{Nd}_{0.8} \mathrm{Sr}_{0.2} \mathrm{NiO}_{2}$ [1]. The nickelate superconductors have similar crystal structure as cuprates, and the monovalent $\mathrm{Ni}^{1+}$-ions also possess the same $3 d^{9}$ configuration as $\mathrm{Cu}^{2+}$-ions. It is therefore thought to be the same as cuprates. However, the parent compound $\mathrm{NdNiO}_{2}$ displays metallic behavior at high temperatures with a resistivity upturn below about $70 \mathrm{~K}$, and shows no sign of any magnetic long-range order in the whole measured temperature range [27]. Similar results have also been found previously in $\mathrm{LaNiO}_{2}$ [28]. These experimental observations are in sharp contrast with the naive expectation of a Mott insulator with AF long-range order for the parent compounds of nickelates. It is therefore important to address what is the nature of the parent compounds and how the AF long-range order is suppressed.

Key experimental evidences. - Figure 1 presents the resistivity and Hall data as functions of temperature for both parent compounds $\mathrm{NdNiO}_{2}$ and $\mathrm{LaNiO}_{2}$. Surprisingly, when the data were put on a linear-log scale, we find that the resistivity $\rho$ upturn well obeys a logarithmic temperature $(\ln T)$ dependence below about 40 $\mathrm{K}$ down to $4 \mathrm{~K}$ for $\mathrm{NdNiO}_{2}$ and below about $70 \mathrm{~K}$ down to $11 \mathrm{~K}$ for $\mathrm{LaNiO}_{2}$. This is a clear evidence of magnetic Kondo scattering [29, 30].

This Kondo scenario is further supported by the Hall effect data in the both compounds. While the Hall coefficient $R_{H}$ exhibits non-monotonic temperature dependence, very different from that of the resistivity in the high temperature metallic regime, it shows the same $\ln T$ dependence at low temperatures. In the Kondo systems, we have $R_{H} \propto \rho$, due to the incoherent skew scattering associated with the localized magnetic impurity [31, 32]. Thus both the resistivity and Hall coefficient support the presence of the magnetic Kondo scattering in the parent compounds of nickelate superconductors. Moreover, at high temperatures where the skew scattering is negligible and the normal Hall effect dominates, the magnitude of the Hall coefficient is found to be only about $-4 \times 10^{-3} \mathrm{~cm}^{3} \mathrm{C}^{-1}$ for $\mathrm{NdNiO}_{2}$ and $-3 \times 10^{-3} \mathrm{~cm}^{3} \mathrm{C}^{-1}$ 

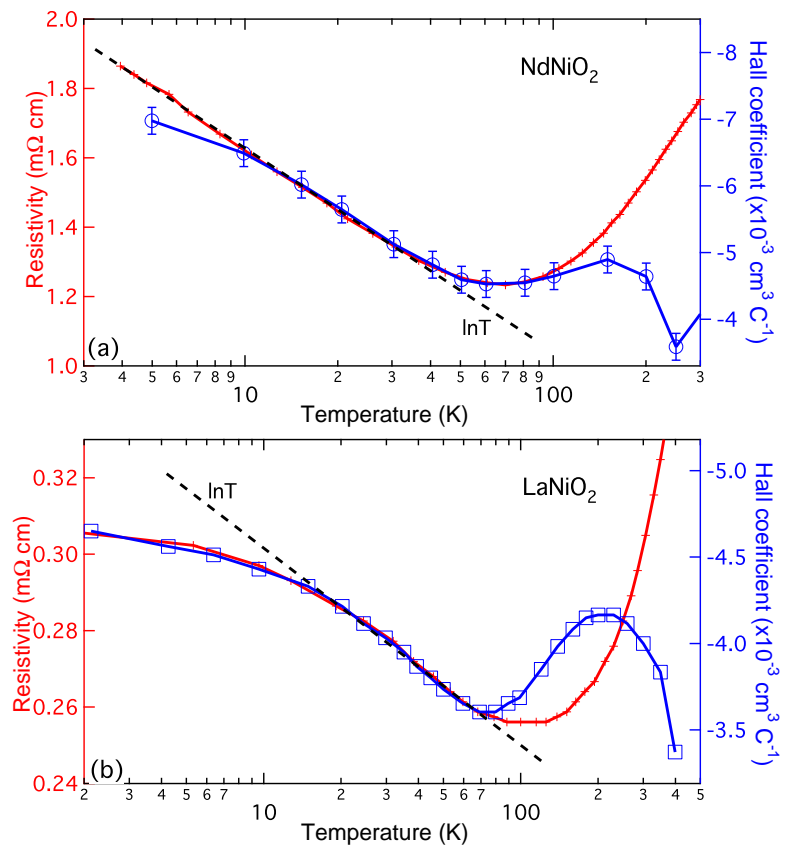

FIG. 1: Logarithmic temperature dependence of the resistivity (red color) and the Hall coefficient (blue color) at low temperatures for (a) $\mathrm{NdNiO}_{2}$ with the experimental data adopted from Ref. [1]; (b) $\mathrm{LaNiO}_{2}$ reproduced from Ref. [28]. The dashed lines are the $\ln T$ fits.

for $\mathrm{LaNiO}_{2}$. Both are an order of magnitude higher than those of normal heavy fermion metals. For example, we have $R_{H} \approx-3.5 \times 10^{-4} \mathrm{~cm}^{3} \mathrm{C}^{-1}$ in all three $\mathrm{Ce} M \mathrm{In}_{5}$ compounds $(M=\mathrm{Co}, \mathrm{Rh}, \mathrm{Ir})$ at high temperatures [33]. This indicates that there are only a few percent of electron-like carriers per unit cell in both $\mathrm{NdNiO}_{2}$ and $\mathrm{LaNiO}_{2}$. Therefore, the parent compounds of nickelates belong to a Kondo system with low-density charge carriers.

Below we examine the Kondo scenario for $\mathrm{NdNiO}_{2}$ from the microscopic picture. The first-principles band structure calculations [34] show that the Nd $5 d$ orbitals in $\mathrm{NdNiO}_{2}$ are hybridized with the Ni $3 d$ orbitals, leading to small Fermi pockets of dominantly Nd $5 d$ electrons in the Brillouin zone. Nd-5d conduction electrons have a low electron density of $n_{c} \ll 1$ per $\mathrm{Ni}$-site, coupling to the localized $\mathrm{Ni}^{1+}$ spin- $1 / 2$ of $3 d_{x^{2}-y^{2}}$ orbital to form Kondo spin singlets (doublons) 35]. Here we have considered Ni-3 $3 d_{x^{2}-y^{2}}$ electrons to be strongly correlated with a large on-site Coulomb repulsion $U$ to disfavor double occupation on the same sites.

With this picture in mind, it is attempted to propose a Kondo Hamiltonian to describe the parent compounds of nickelates. However, unlike the usual Kondo lattice model, the $\mathrm{Ni}^{1+}$ localized spins here are coupled mainly by superexchange interaction through the $\mathrm{O}-2 p$ orbitals as same as in the cuprates, though the coupling on nickel

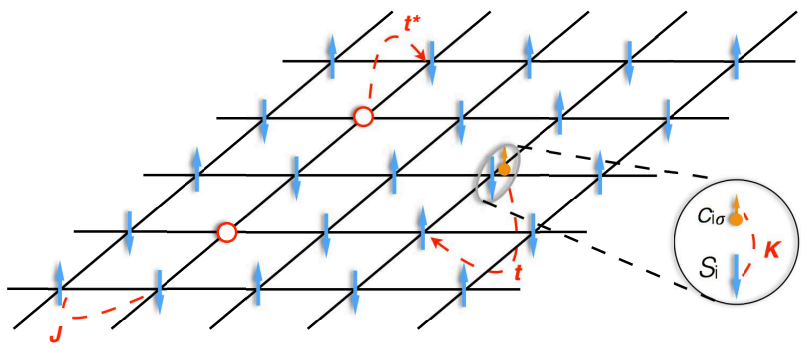

FIG. 2: Illustration of the effective model on a twodimensional square lattice of $\mathrm{NiO}_{2}$ plane of $\mathrm{NdNiO}_{2}$. Blue arrow represents $\mathrm{Ni}$-spin, which interacts with its neighboring spin antiferromagetically by coupling $J$. Orange arrow denotes Nd-5d electron, which couples to Ni-spin by the Kondo coupling $K$, to form a Kondo singlet (doublon). Red circle represents $\mathrm{Ni}-3 d^{8}$ configuration, or a holon. $t$ and $t^{*}$ are the hoping integrals of doublon and holon, respectively. Not shown is the holon-doublon anhilation into a Ni-spin.

sites is small. Thus the starting point should actually be a background lattice of $\mathrm{Ni}^{1+}$ localized spins with the nearest-neighbor AF Heisenberg superexchange coupling and additional local Kondo exchanges with the itinerant $5 d$ electrons.

For the parent compound, we have correspondingly $1-n_{c}$ electrons per Ni-site, or $n_{c} N_{s}\left(N_{s}\right.$ as the total number of Ni-sites) empty nickel sites (holons) on the $\mathrm{NiO}_{2}$ plane. This introduces a strongly renormalized hopping term of holons. A schematic picture is displayed in Fig.2 The presence of both the Kondo singlets/doublons and the holons can suppress very efficiently the AF long-range order and cause a phase transition from the Mott insulating state to a metallic state. Actually, as we will show below, an effective low-energy model Hamiltonian can be derived in terms of the doublons, holons and localized spins, describing a self-doped Mott metallic state even in the parent $\mathrm{LnNiO}_{2}(\mathrm{Ln}=\mathrm{La}, \mathrm{Nd})$ compounds. Upon further Sr hole doping, such a low-energy effective model is expected to exhibit $d$-wave pairing instability as in the usual $t$ - $J$ model.

Effective model Hamiltonian. - We consider Ni$3 d^{8}$ and Nd-5 $d^{0}$ as the vacuum, and start with the localized $3 d_{x^{2}-y^{2}}$ spins on the $\mathrm{NiO}_{2}$ plane that form a two-dimensional quantum Heisenberg model with nearest neighbour AF superexchange interactions,

$$
H_{J}=J \sum_{\langle i j\rangle} S_{i} \cdot S_{j}
$$

This is similar to the cuprates, where the superexchange interaction is induced by the $\mathrm{O}-2 p$ orbitals and the parent compound is a Mott insulating state with AF long-range orders. In nickelates, however, we have to further consider the Kondo coupling with the Nd or La $5 d$ conduction electrons. This leads to the following Kondo lattice 
Hamiltonian,

$$
H_{K}=-t \sum_{\langle i j\rangle, \sigma}\left(c_{i \sigma}^{\dagger} c_{j \sigma}+h . c .\right)+\frac{K}{2} \sum_{j \alpha ; \sigma \sigma^{\prime}} S_{j}^{\alpha} c_{j \sigma}^{\dagger} \tau_{\sigma \sigma^{\prime}}^{\alpha} c_{j \sigma^{\prime}},
$$

where $t$ describes the effective hoping amplitude of the $5 d$ itinerant electrons projected on the square lattice sites of the $\mathrm{Ni}^{1+}$ ions, and $\tau^{\alpha}(\alpha=x, y, z)$ are the spin- $1 / 2$ Pauli matrices. We consider a single $5 d$ orbital for $\mathrm{Nd}$ for simplicity. For a low-carrier density system, the average number of conduction electrons is very small, i.e., $N_{s}^{-1} \sum_{j \sigma}\left\langle c_{j \sigma}^{\dagger} c_{j \sigma}\right\rangle=n_{c} \ll 1$.

In the parent compound $\mathrm{LnNiO}_{2}(\mathrm{Ln}=\mathrm{La}, \mathrm{Nd})$, the total electron density is 1 per unit cell, hence the total holon density $n_{h}=n_{c}$. For Sr doped compounds, we have $\delta=n_{h}-n_{c}>0$. To describe the doping effect, we introduce the pseudofermion representation for the spin- $1 / 2$ local moments,

$$
S_{j}^{+}=f_{j \uparrow}^{\dagger} f_{j \downarrow}, S_{j}^{-}=f_{j \downarrow}^{\dagger} f_{j \uparrow}, S_{j}^{z}=\frac{1}{2}\left(f_{j \uparrow}^{\dagger} f_{j \uparrow}-f_{j \downarrow}^{\dagger} f_{j \downarrow}\right),
$$

where $f_{j \sigma}$ is a fermionic operator and denotes a spinon on site $j$. The holon hopping term between empty nickel sites is then given by

$$
H_{t^{*}}=-t^{*} \sum_{\langle i j\rangle, \sigma}\left(h_{i} f_{i \sigma}^{\dagger} f_{j \sigma} h_{j}^{\dagger}+\text { h.c. }\right),
$$

where $h_{j}^{\dagger}$ is the bosonic operator creating a holon on the $j$-th site. In this representation, the Ni $3 d_{x^{2}-y^{2}}$ electron operator is given by $d_{j \sigma}=h_{j}^{\dagger} f_{j \sigma}$ with a local constraint, $h_{j}^{\dagger} h_{j}+\sum_{\sigma} f_{j \sigma}^{\dagger} f_{j \sigma}=1$. This is just the slave-boson representation for the constrained electrons without double occupancy.

All together, the total model Hamiltonian for nickelates consists of three terms,

$$
H=H_{J}+H_{K}+H_{t^{*}} .
$$

This model contains several key energy scales. While the electron hopping $t$ may be roughly estimated from band calculations, the holon hopping $t^{*}$ is strongly renormalized due to the background AF correlations and thus contribute little to the transport measurements in the parent compounds. The kinetic energy in the Hamiltonian is therefore relatively small due to the small number of charge carriers without Sr doping. The Heisenberg superexchange $J$ is also expected to be smaller (possibly the order of $10 \mathrm{meV}$ ) compared to that (about $100 \mathrm{meV}$ ) in cuprates due to the larger charge transfer energy between O- $2 p$ and Ni-3 $d_{x^{2}-y^{2}}$ orbitals. Actually the Heisenberg exchange energy is further reduced in a paramagnetic background. For the Kondo temperature of the value of $10 \mathrm{~K}$ or $1 \mathrm{meV}$, which is about one tenth of the temperature of resistivity minimum in both $\mathrm{LaNiO}_{2}$ and $\mathrm{NdNiO}_{2}$, a Kondo coupling of roughly the order of $100 \mathrm{meV}$ would be expected for a low-carrier density system with a small electron density of states [37]. Thus for the parent compounds of nickelates, the Kondo coupling is a relatively large energy scale in the above model Hamiltonian.

From these analyses, one may anticipate that the ground state of the nickelate parent compounds may be to some extent captured by the large $K$ limit of the Hamiltonian. The Kondo singlets are then well established between the Ni $3 d_{x^{2}-y^{2}}$ spins and the $5 d$ conduction electrons. To explore this possibility, we introduce the doublon operators for the on-site Kondo spin singlet and triplets:

$$
\begin{aligned}
& b_{j 0}^{\dagger}=\frac{1}{\sqrt{2}}\left(f_{j \uparrow}^{\dagger} c_{j \downarrow}^{\dagger}-f_{j \downarrow}^{\dagger} c_{j \uparrow}^{\dagger}\right) ; \\
& b_{j 1}^{\dagger}=f_{j \uparrow}^{\dagger} c_{j \uparrow}^{\dagger}, b_{j 2}^{\dagger}=\frac{1}{\sqrt{2}}\left(f_{j \uparrow}^{\dagger} c_{j \downarrow}^{\dagger}+f_{j \downarrow}^{\dagger} c_{j \uparrow}^{\dagger}\right), b_{j 3}^{\dagger}=f_{j \downarrow}^{\dagger} c_{j \downarrow}^{\dagger} .
\end{aligned}
$$

The Kondo exchange term is then transformed to,

$$
\frac{K}{2} \sum_{j \alpha ; \sigma \sigma^{\prime}} S_{j}^{\alpha} c_{j \sigma}^{\dagger} \tau_{\sigma \sigma^{\prime}}^{\alpha} c_{j \sigma^{\prime}}=\frac{K}{4} \sum_{\mu=1}^{3} b_{j \mu}^{\dagger} b_{j \mu}-\frac{3 K}{4} \sum_{j} b_{j 0}^{\dagger} b_{j 0},
$$

which describes the doublon formation on each site, namely, the Kondo singlet or triplet pair formed by each conduction electron with the localized spinon. However, the Kondo triplet costs a larger energy of $K$ and is therefore not favored. In addition, there can also be three-electron states with two conduction electrons and the localized spinon on the same site, $e_{j \sigma}^{\dagger}=f_{j \sigma}^{\dagger} c_{j \uparrow}^{\dagger} c_{j \downarrow}^{\dagger}$, and one-electron states with the unpaired spinon only, $\widetilde{f}_{j \sigma}=\left(1-n_{j}^{c}\right) f_{j \sigma}$. So these operators should be used with the constraint

$$
h_{j}^{\dagger} h_{j}+\sum_{\mu=0}^{3} b_{j \mu}^{\dagger} b_{j \mu}+\sum_{\sigma}\left(\widetilde{f}_{j \sigma}^{\dagger} \widetilde{f}_{j \sigma}+e_{j \sigma}^{\dagger} e_{j \sigma}\right)=1,
$$

for each site. These new operators do not commute in a simple way so for simplicity we should avoid direct operation using their commutation relations.

In the large $K$ limit, following the method used in Ref. [36, 38], a low-energy effective Hamiltonian can be derived by first rewriting the hopping term $H_{t}$ in terms of the new operators $b_{j 0}, b_{j \mu}(\mu=1,2,3), e_{j \sigma}$, and $\widetilde{f}_{j \sigma}$ and then employing the canonical transformation, $H_{\text {eff }}=e^{-S} H e^{S}$, to eliminate all high-energy terms containing $b_{j \mu}(\mu=1,2,3)$ and $e_{j \sigma}$ while keeping only the on-site doublon $\left(b_{j 0}\right)$ and unpaired spinons $\left(\widetilde{f}_{j \sigma}\right)$. In the infinite- $K$ limit, in particular, the low-energy effective model becomes a simple form

$$
\begin{aligned}
H_{\mathrm{eff}}= & -t^{*} \sum_{\langle i j\rangle, \sigma}\left(h_{i} \widetilde{f}_{i \sigma}^{\dagger} \widetilde{f}_{j \sigma} h_{j}^{\dagger}+\text { h.c. }\right)+J \sum_{\langle i j\rangle} \widetilde{S}_{i} \cdot \widetilde{S}_{j} \\
& -\frac{t}{2} \sum_{\langle i j\rangle, \sigma}\left(b_{i 0}^{\dagger} \widetilde{f}_{i \sigma} \widetilde{f}_{j \sigma}^{\dagger} b_{j 0}+\text { h.c. }\right)
\end{aligned}
$$


where the spin operators are expressed as $\widetilde{S}_{j}^{\alpha}=$ $\sum_{\sigma \sigma^{\prime}} \tilde{f}_{j \sigma}^{\dagger} \frac{1}{2} \tau_{\sigma \sigma^{\prime}}^{\alpha} \tilde{f}_{j \sigma^{\prime}}$ with a local constraint $h_{j}^{\dagger} h_{j}+b_{j 0}^{\dagger} b_{j 0}+$ $\sum_{\sigma} \widetilde{f}_{j \sigma}^{\dagger} \widetilde{f}_{j \sigma}=1$. For a large but finite $K$, apart from some complicated interactions, an additional term should be included

$$
H_{b}=-\frac{3}{4}\left(K+\frac{t^{2}}{K}\right) \sum_{j} b_{j 0}^{\dagger} b_{j 0}+\frac{5 t^{2}}{12 K} \sum_{\langle i j\rangle} b_{i 0}^{\dagger} b_{i 0} b_{j 0}^{\dagger} b_{j 0},
$$

which could be used to describe the doublon condensation.

Discussions - The above effective low-energy Hamiltonian is very similar to the usual $t$ - $J$ model for cuprates [12], except that it includes two different types of charge carriers: the Kondo singlets (doublons) and the holons on the Ni sites. Their presence can efficiently suppress the AF long-range order and bring the phase transition from a Mott insulator to a self-doped Mott metallic state. The effective model therefore describes a self-doped Mott insulating state as the parent state of nickelate superconductors, with possibly an enhanced effective mass for the charge carriers. It also provides an interesting example of holon-doublon excitations for destroying the Mott insulator, although the doublons here are associated with the Kondo singlets rather than doubly occupied $\mathrm{Ni} 3 d_{x^{2}-y^{2}}$ orbitals. At high temperatures, the doublons become deconfined, causing incoherent Kondo scattering as observed in experiments.

Furthermore, the Sr hole doping reduces the number of electron carriers and thus suppresses the contribution of doublons. At large doping, the effective model is then reduced to the usual $t$ - $J$ model. In cuprates, the $\mathrm{Cu}$ $3 d_{x^{2}-y^{2}}$ orbitals and the O- $2 p$ orbitals are strongly hybridized. The doped holes sit on the oxygen sites, forming the Zhang-Rice singlets with $\mathrm{Cu}^{2+}$ localized spins. By contrast, the holes in nickelates reside on the Ni-ions, leading to a spin zero state or holon due to the much less overlap with the $\mathrm{O}-2 p$ band [3]. Sr doping hence introduces extra holes on the Ni sites, which further drives the system away from the AF Mott insulating phase, resembling that in the optimal or overdoped cuprates. However, even at $20 \% \mathrm{Sr}$ doping, the electron carriers are still present, as manifested by the negative Hall coefficient at high temperatures in $\mathrm{Nd}_{0.8} \mathrm{Sr}_{0.2} \mathrm{NiO}_{2}$ [1]. Since the electron carrier density is reduced with hole doping, the smaller magnitude of $R_{H}$ in $\mathrm{Nd}_{0.8} \mathrm{Sr}_{0.2} \mathrm{NiO}_{2}$ cannot be explained by a single carrier model but rather indicates a cancellation of electron and hole contributions. The latter grows gradually with Sr doping and eventually becomes dominant at low temperatures in $\mathrm{Nd}_{0.8} \mathrm{Sr}_{0.2} \mathrm{NiO}_{2}$, causing the sign change of the Hall coefficient below about $50 \mathrm{~K}$.

Experimentally, with $20 \% \mathrm{Sr}$ doping in $\mathrm{NdNiO}_{2}$, superconductivity also emerges and has the highest transition temperature of about $15 \mathrm{~K}$. Interestingly, when fitted with a power-law temperature dependence, $\rho \propto T^{\alpha}$, we notice for this particular sample that the electric resistivity exhibits a non-Fermi liquid behavior in the normal state. Actually, an excellent agreement could be obtained with $\alpha=1.13 \pm 0.02$ over a wide range from slightly above the superconducting transition temperature up to the room temperature. In fact, for all reported samples with high superconducting transition temperature, a good power-law fit can always be obtained with $\alpha \approx 1.1 \sim 1.3$. This is reminiscent to the optimal doped cuprate superconductors and suggests a similar strange metal phase for the normal state of optimal doped nickelate superconductors.

Conclusion and outlook - Our proposed model bridges the Kondo lattice model for heavy fermions and the $t-J$ model for cuprates. However, it is different from both models in the sense that it combines some new physics that is not included in either of them. Unlike the usual Kondo lattice system, the exchange interaction here between localized spins are produced by the superexchange coupling rather than the RKKY coupling. Thus at low carrier density, the magnetic ground state is not ferromagnetic as one would expect for the Kondo lattice. On the other hand, the nickelate system indeed exhibits incoherent Kondo scatterings as revealed in the transport properties at high temperatures. Unlike cuprates, the presence of strong Kondo coupling could lead to holondoublon excitations even in the parent compound. This self-doping effect suppresses the AF long-range order and produces the paramagnetic metallic ground state. The parent compound of nickelate superconductor is therefore described as a self-doped Mott state. This makes it somehow different from the cuprates but resembles certain organic superconductors under pressure, which reduces the on-site Coulomb repulsion $U$ and induces a transition from Mott insulator to gossamer superconductor with both holons and doublons [39].

Acknowledgment.- The authors would like to acknowledge the discussions with Wei-Qiang Chen, Hong-Ming Weng, and $\mathrm{Yu} \mathrm{Li}$. This work was supported by the National Key Research and Development Program of MOST of China (2016YFYA0300300, 2017YFA0302902, 2017YFA0303103), the National Natural Science Foundation of China (11774401 and 11674278), the State Key Development Program for Basic Research of China (2014CB921203 and 2015CB921303), and the Strategic Priority Research Program of CAS (Grand No. XDB28000000).

\footnotetext{
* gmzhang@tsinghua.edu.cn

† yifeng@iphy.ac.cn

$\ddagger$ fuchun@ucas.ac.cn
}

[1] D. Li, K. Lee, B. Y. Wang, M. Osada, S. Crossley, H. R. Lee, Y. Cui, Y. Hikita, and H. Y. Hwang, Nature 572, 
624 (2019).

[2] A. S. Botana and M. R. Norman, arXiv:1908.10946 (2019).

[3] M. Jiang, M. Berciu, and G. Sawatzky, arXiv:1909.02557 (2019).

[4] H. Sakakibara, H. Usui, K. Suzuki, T. Kotani, H. Aoki, and K. Kuroki, arXiv:1909.00060 (2019).

[5] M. Hepting, D. Li, C. J. Jia, H. Lu, E. Paris, Y. Tseng, X. Feng, M. Osada, E. Been, Y. Hikita, Y.-D. Chuang, Z. Hussain, K. J. Zhou, A. Nag, M. Garcia-Fernandez, M. Rossi, H. Y. Huang, D. J. Huang, Z. X. Shen, T. Schmitt, H. Y. Hwang, B. Moritz, J. Zaanen, T. P. Devereaux, and W. S. Lee, arXiv:1909.02678 (2019).

[6] Y. Normura, M. Hirayama, T. Tadano, Y. Yoshimoto, K. Nakamura, and R. Arita, arXiv:1909.03942 (2019).

[7] J. Gao, Z. Wang, C. Fang, and H. Weng, arXiv:1909.04657 (2019).

[8] J. P. Bednorz and K. A. Muller, Z. Phys. B 64, 189 (1986).

[9] P. W. Anderson, Science 235, 1196 (1987).

[10] P. W. Anderson, P. A. Lee, M. Randeria, T. M. Rice, N. Trivedi, and F. C Zhang, J. Phys.: Condens. Matter 16, R755 (2004).

[11] P. A. Lee, N. Nagaosa, and X. G. Wen, Rev. Mod. Phys. 78, 17 (2006).

[12] F. C. Zhang, and T. M. Rice, Phys. Rev. B 37, 3759 (1988).

[13] Z. X. Shen, D. S. Dessau, B. O. Wells, D. M. King, W. E. Spicer, A. J. Arko, D. Marshall, L. W. Lombardo, A. Kapitulnik, P. Dickinson, S. Doniach, J. DiCarlo, T. Loeser, and C. H. Park, Phys. Rev. Lett. 70, 1553 (1993).

[14] D. A. Wollman, D. J. Van Harlingen, W. C. Lee, D. M. Ginsberg, and A. J. Leggett, Phys. Rev. Lett. 71, 2134 (1993).

[15] C. C. Tsuei, J. R. Kirtley, C. C. Chi, L. S. Yujahnes, A. Gutpa, T. Shaw, J. Z. Sun, and M. B. Ketchen, Phys. Rev. Lett. 73, 593 (1994).

[16] V. I. Anisimov, D. Bukhvalov, and T. M. Rice, Phys. Rev. B 59, 7901-7906 (1999).

[17] M. A. Hayward, M. A. Green, M. J. Rosseinsky, and J. Sloan, J. Am. Chem. Soc. 121, 8843 (1999).

[18] K.-W. Lee and W. E. Pickett, Phys. Rev. B 70, 165109 (2004).

[19] A. S. Botana, V. Pardo, and M. R. Norman, Phys. Rev. Materials 1, 021801(R) (2017).

[20] J. Chaloupka and G. Khaliullin, Phys. Rev. Lett. 100, 016404 (2008).

[21] P. Hansmann, X. Yang, A. Toschi, G. Khaliullin, O. K. Andersen, and K. Held, Phys. Rev. Lett. 103, 016401 (2009).

[22] S. Middey, J. Chakhalian, P. Mahadevan, J. W. Freeland, A. J. Millis, and D. D. Sarma, Annu. Rev. Mater. Res. 46, 305 (2016).

[23] A. V. Boris, Y. Matiks, E. Benckiser, A. Frano, P.
Popovich, V. Hinkov, P. Wochner, M. Castro-Colin, E. Detemple, V. K. Malik, C. Bernhard, T. Prokscha, A. Suter, Z. Salman, E. Morenzoni, G. Cristiani, H.-U. Habermeier, and B. Keimer, Science 332, 937 (2011).

[24] E. Benckiser, et. al., E. Benckiser, M. W. Haverkort, S. Brück, E. Goering, S. Macke, A. Frañó, X. Yang, O. K. Andersen, G. Cristiani, H.-U. Habermeier, A. V. Boris, I. Zegkinoglou, P. Wochner, H. Kim, V. Hinkov, and B. Keimer, Nat. Mater. 10, 189 (2011).

[25] A. S. Disa, D. P. Kumah, A. Malashevich, H. Chen, D. A. Arena, E. D. Specht, S. Ismail-Beigi, F. J. Walker, and C. H. Ahn, Phys. Rev. Lett. 114, 026801 (2015).

[26] J. Zhang, A. S. Botana, J. W. Freeland, D. Phelan, H. Zheng, V. Pardo, M. R. Norman, and J. F. Mitchell, Nat. Phys. 13, 864 (2017).

[27] M. A. Hayward and M. J. Rosseinsky, Solid State Sciences 5, 839 (2003).

[28] A. Ikeda, Y. Krockenberger, H. Irie, M. Naito, and H. Yamamoto, Applied Physics Express 9, 061101 (2016).

[29] A. C. Hewson, The Kondo Problem to Heavy Fermions, Cambridge University Press, 1993.

[30] Actually an alternative explanation for the resistivity upturn at low temperatures is weak localization due to the presence of disorder holes in the $\mathrm{NiO}_{2}$ plane. It can also give rise to a logarithmic temperature dependent correction. However, the corresponding correction to the Hall coefficient is independent of temperature in the same region, which does not support this explanation.

[31] A. Fert and P. M. Levy, Phys. Rev. Lett. 36, 1907 (1987).

[32] N. Nagaosa, J. Sinova, S. Onoda, A. H. MacDonald, and N. P. Ong, Rev. Mod. Phys. 82, 1539 (2010).

[33] M. F. Hundley, A. Malinowski, P. G. Pagliuso, J. L. Sarrao, and J. D. Thompson, Phys. Rev. B 70, 035113 (2004).

[34] K. W. Lee and W. E. Pickett, Phys. Rev. B 70, 165109 (2004).

[35] The presence of magnetic impurities may be at first glance ascribed to the $\mathrm{Nd} 4 f$ moments. However, the $\mathrm{Nd}^{3+}$ ion contains three $f$ electrons forming a localized spin-3/2 moment, which acts more like a classical spin as in manganites and therefore disfavors spin-flip scattering as the quantum spin-1/2 moment. Their energy level is also far away from the Fermi energy, so it is reasonable to ignore the $\mathrm{Nd} 4 f$ electrons.

[36] C. Lacroix, Solid State Commun. 54, 991 (1985).

[37] Y. F. Yang, Z. Fisk, H. O. Lee, J. D. Thompson, and D. Pines, Nature 454, 611 (2008).

[38] M. Sigrist, K. Ueda, and H. Tsunetsugu, Phys. Rev. B 46, 175 (1992).

[39] R. B. Laughlin, cond-mat/0209269 F. C. Zhang, Phys. Rev. Lett. 90, 207002 (2003); B. A. Bernevig, R. B. Laughlin, and D. I. Santiago, Phys. Rev. Lett. 91, 147003 (2003). 